\title{
Solitary Fibrous Tumor of the Kidney with Massive Retroperitoneal Recurrence. A Case Presentation
}

\author{
Sfoungaristos S., Papatheodorou M., Kavouras A., Perimenis P. \\ Department of Urology, University Hospital of Patras, Patras, Greece \\ Received January 7, 2012; Accepted June 25, 2012.
}

Key words: Solitary fibrous tumor - Kidney - Recurrence - Malignant

\begin{abstract}
Solitary fibrous tumor is an unusual spindle cell neoplasm that usually occurs in the pleura but has also been described in other sites. Renal presentation is rare and only 38 cases of SFT of the kidney have been described until now. Up to $90 \%$ of the tumors have benign characteristics. Local retroperitoneal recurrence is extremely rare. We report a case of a large malignant solitary fibrous tumor recurrence after the surgical excision of the primary tumor. Histological examination of the specimen confirmed the diagnosis by revealing highly positive reaction of the neoplasmatic cells for vimentin, CD34, bcl-2 and moderately positive for actin. The rate of Ki67 lied between $2-7 \%$. No adjuvant therapy was given to the patient and he is disease-free with no imaging findings of recurrence or metastasis 9 months after the re-operation.
\end{abstract}

Mailing Address: Stavros Sfoungaristos, MD., Department of Urology, University Hospital of Patras, Building A, 4th Floor, Rion, 26500 Patras, Greece; Phone: +302 610999 367; Fax: +302 610993 981; e-mail: sfoungaristosst@gmail.com 


\section{Introduction}

Solitary fibrous tumor (SFT) is an uncommon type of neoplasm of mesenchymal origin, most frequently arising in the pleura. Other extrapleural locations include the orbit, paranasal sinuses, nasal cavity, upper respiratory tract, lung, major salivary glands, breasts, meninges, liver and urogenital organs. SFT of the kidney is rare and up to date only few cases have been reported. Morphologically, SFT is characterized by spindle cell proliferation with a patternless architecture, and the final diagnosis is based on immunohistochemistry (Wang et al., 2001; Gengler and Guillou, 2006).

In the present report, we describe a case of renal SFT with a rather impressive local retroperitoneal recurrence 3 years after the primary nephrectomy.

\section{Case report}

A 72-year-old man was admitted to our hospital with one-month history of mild pain in his left flank, without gross hematuria or other constitutional symptoms. Physical examination revealed a palpable right flank mass. Laboratory data of blood and urine, including cytology, were within normal limits. A computed tomography (CT) scan of the abdomen showed a well-delineated mass arising from the upper pole of left kidney measuring $7 \times 6 \mathrm{~cm}$ with contrast enhancement. The patient underwent left radical nephrectomy with a presumptive diagnosis of renal cell carcinoma (RCC). The pathologic diagnosis of the nephrectomy specimen was that of a solitary fibrous tumor with negative surgical margins and no infiltration of the perinephric fat or Gerota's fascia. Neither chemotherapy nor radiation therapy



Figure 1 - Solitary fibrous tumor in the retroperitoneum. 


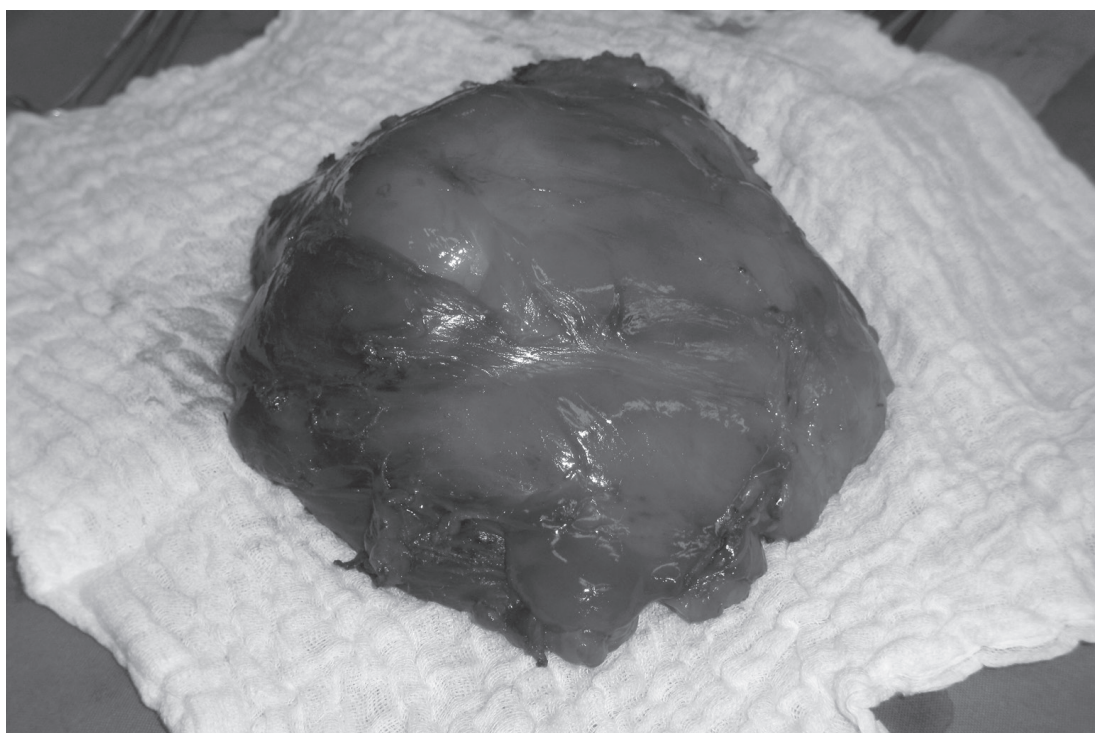

Figure 2 - The first part of the excised tumor.

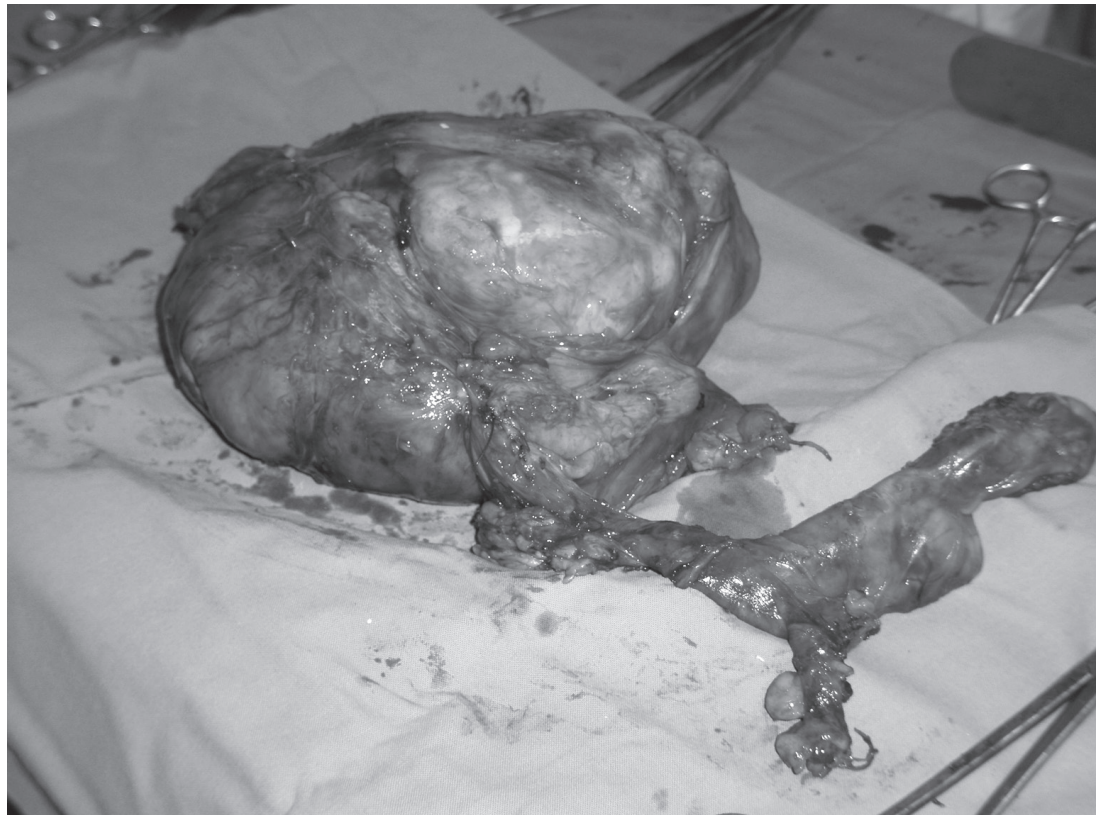

Figure 3 -The second part of the excised tumor.

Sfoungaristos S.; Papatheodorou M.; Kavouras A.; Perimenis P. 
was given postoperatively. After a negative first year of follow up schedule with biannual abdominal CT scan and quarterannual ultrasound, chest $\mathrm{X}$-ray, blood biochemistry and physical examination, the patient was lost in the second year. $\mathrm{He}$ re-appeared in the third year complaining for mild pain in the left abdomen. A CT scan was performed and revealed local recurrence of a mass measuring $12 \times 11 \mathrm{~cm}$ with contrast enhancement. A complete surgical resection was performed and the pathologic assessment confirmed the recurrence of a solitary fibrous tumor (Figures 1-3). Thereafter the patient follows a strict algorithm for surveillance, being well without evidence of recurrence or metastasis 9 months after the re-operation.

\section{Pathological findings}

The pathologic review revealed a neoplasm of mesenchymal origin with morphologic and immunohistochemical characteristics in consistence with those of solitary fibrous tumor, as already mentioned in the patient's history.

The examination of the first extract of the tumor demonstrated hypercellularity and diffuse nuclear atypia, combined with focal areas of necrosis and hemorrhage, while the mitotic rate was at 3-7/10. The above data, combined with the patient's history, favor the malignant type of neoplasmatic behavior. Similar histological characteristics were also present at the primary tumor examination. Actually, hypercellularity, diffuse nuclear atypia with focal necrosis and hemorrhage and a mitotic rat e of $2-5 / 10$ were reported. The size of the first extract was measured at $20 \times 19 \times 10 \mathrm{~cm}$, weighed 1,852.5 g and was enclosed in a pseudocapsule. Its substance was mostly white, sometimes red, appeared nodular and at parts rough.

The second extract was also surrounded by a pseudocapsule, but was remarkably smaller at $16 \times 14 \times 6 \mathrm{~cm}$ and weighing $634 \mathrm{~g}$. It appeared fibroelastic in substance, white, with a dense surface.

Both specimens were therefore immunohistochemically analyzed for their reactivity towards keratin, AE1, AE3, vimentin, SMA, actin, desmin, S100, CD31, CD34, CD99, bcl-2, c-kit and Ki67. The neoplasmatic cells were highly positive for vimentin, CD34, bcl-2 and moderately positive for actin. On the contrary, the tumor demonstrated negative reactivity for SMA, S100, CD31, CD99 and CD117 (c-kit). The rate of $\mathrm{Ki67}$ lied between $2-7 \%$.

The diagnosis of SFT was therefore confirmed once more, and to be specific, a rare case of a recurrent and malignant SFT.

\section{Discussion}

SFT is an uncommon type of neoplasm. Gelb et al. (1996) was the first to describe in 1996 a SFT arising from the kidney. Since then, only 38 cases have been described, categorizing renal SFT as extremely rare. The renal capsule has been considered as the most usual site of origin.

Renal SFTs can be easily confused clinically with carcinomas or sarcomas and the diagnosis is set postoperatively (Hirano et al., 2009). Pathologic analysis shows 
spindle cell proliferation in a patternless architecture, combined with alternating hypocellular and hypercellular areas. There may be different characteristics between focal areas of the same tumor but in general the spindle cells are infiltrated with bands of collagen and in some cases with hemangiopericytoma-like vessels. Immunohistochemistry sets the final diagnosis. The profile of the tumor is highly characteristic, although not entirely specific. SFTs reveal diffusely positive reactivity for CD34 which is now considered to be a characteristic marker of SFT. Other positive markers found frequently in SFTs include Bcl-2 and CD99. On the contrary, SFTs in general show negative reactivity for cytokeratin, alpha-SMA, S-100, CD31 and c-kit. These markers contribute to the differential diagnosis of SFTs from other spindle cell tumors.

Concerning the therapeutic options, complete surgical excision of the lesion seems to be the gold standard so far (Morimitsu et al., 2000). Incomplete resection is one of the most important factors related to poor prognosis of SFT. To date there isn't any data on the role of adjuvant radiation or chemotherapy in the treatment, neither primarily or of a recurrence, as the tumor seems chemoradio resistant. The sole treatment applied in our case was also complete surgical excision, with the patient remaining free of disease 9 months after the reoperation for the recurrence.

Following complete surgical resection, most of the patients show no evidence of disease during follow-up. However, follow-up is mandatory, because malignancy, recurrence, or both have been reported in up to 10-15\% of the cases. In our case, local recurrence of a renal SFT, with dimensions even bigger than the primary lesion, was reported in a rather short period of time after surgical resection. Additionally, the clinical behavior cannot be accurately predicted on histopathological basis with benign-appearing tumors exhibiting aggressive behavior and vice versa. Therefore, all patients with these tumors need to be on long-term follow-up (Znati et al., 2007).

\section{References}

Gelb, A. B., Simmons, M. L., Weidner, N. (1996) Solitary fibrous tumor involving the renal capsule. Am. J. Surg. Pathol. 20,1288-1295.

Gengler, C., Guillou, L. (2006) Solitary fibrous tumour and haemangiopericytoma: evolution of a concept. Histopathology 48, 63-74.

Hirano, D., Mashiko, A., Murata,Y., Satoh, K., Ichinose, T., Takahashi, S., Jike, T., Sugitani, M. (2009) A case of solitary fibrous tumor of the kidney: an immunohistochemical and ultrastructural study with a review of the literature. Med. Mol. Morphol. 42, 239-244.

Morimitsu, Y., Nakajima, M., Hisaoka, M., Hashimoto, H. (2000) Extrapleural solitary fibrous tumor: clinicopathologic study of 17 cases and molecular analysis of the p53 pathway. APMIS 108, 617-625.

Wang, J., Arber, D. A., Frankel, K., Weiss, L. M. (2001) Large solitary fibrous tumor of the kidney: report of two cases and review of the literature. Am. J. Surg. Pathol. 25, 1194-1199.

Znati, K., Chbani, L., El Fatemi, H., Harmouch, T., Kamaoui, I., Tazi, F., Bennis, S., Amarti, A. (2007) Solitary fibrous tumor of the kidney:A case report and review of the literature. Rev. Urol. 9, 36-40. 